rectum, adjacent to the prostatic gland. The irritation rapidly disappeared after the ulcers had been excised or treated with pure nitric acid. This suggests the importance of making a careful rectal examination in these cases.

Dr. BuRCHARD, in closing the discussion, said he has yet to see any disease of the genito-urinary organs that, of itself, is capable of giving rise to distinct and profound nervous disease unless the patient is predisposed either by heredity or some acquired condition. In the three cases presented by him, the patients were all more or less broken down and hysterical.

\title{
ON THE SELF-REGULATION OF THE
} BEAT OF THE HEART.

Dr. S. J. MeLtzer read a paper on this subject. He first reviewed the various theories that have been advanced to explain the mechanism of the heart's action, among them the accumulation of blood in the heart, automatism, etc. All these theories have been disproved, and after centuries of labor and research we stand to-day, with the same apparent helplessness as centuries ago, before the puzzling problem: what makes the heart beat?

Dr. Meltzer said that his theory of the mechanism of the heart may be briefly presented as follows: The heart harbors in some of its anatomical sub-strata two antagonistic functions; namely, the functions of contractibility and of inhibition. These functions belong to the heart itself, and not to the endings of the peripheral nerves. By any stimulus which we may apply to the heart directly or otherwise, we affect both functions simultaneously. During each systole a degree of pressure is developed sufficient to stimulate mechanically both functions at once. But during the stimulation the inhibitory effect prevails and therefore the heart is bound to relax. With the relaxation, however, the stimulation subsides, and we then have before us the period of the after-effects. Here we first see the short period of the inhibitory aftereffect, winding up as a diastolic pause, after which the after-effect of the function for contraction makes its appearance, which means that a contraction of the heart is bound to appear. This contraction would last many minutes if the whole after-effect would be allowed to wind up. But since this contraction means a new systole and a new stimulation, it is then cut off by a newly 
aroused inhibitory effect; therefore, instead of a prolonged contraction, a new cycle of relaxation, diastolic pause, and consequent contraction takes place. In other words, the consequence of each contraction is a cycle of relaxation, diastolic pause and contraction. Thus cach heart beat generates its subsequent diastole and systole, and $w c$ may therefore say that the beats of the heart are regulating their own rhythm.

In conclusion, Dr. Meltzer said that his theory of the self-regulation of the beat of the heart is similar, in the main points, with his theory of the self-regulation of respiration, which he has described elsewhere. Here, as well as there, we have the antagonism between inhibition and contraction; here, as well as there, the inhibition prevails during the stimulation, while the contraction over-lasts in the period of the after-effects; here, as well as there, the pause corresponds to the shortened inhib. itory after-effect, and here, as well as there, the stimulus is a mechanical one, produced by the natural function of the acting organ; here it is the contraction of the heart; there it is the expansion of the lungs. Certainly neither of these theories suffer by their mutual resemblance.

After some remarks by Drs. SACHS and CoLliss, the Society passed a vote of thanks to Dr. Meltzer for his excellent paper.

A Resolution was adopted by the Society, respectfully requesting Governor Flower to grant his approval of the measure passed by the Legislature providing for the establishment of an Epileptic Colony in Livingston County, N. Y.

PHILADELPHIA NEUROLOGICAL SOCIETY. Stated Mccting, April 24, 1893.

The President, Dr. Charles K. Mills, in the chair.

Dr. Wr. J. TAYLOR, presented

\section{A CASE OF DEFORMITY OF LEFT FOOT IN A CHILD ELEVEN MONTHS OF AGE.}

The family history is perfectly good in every respect, d except the deformity of the left foot, for which she brought here to-night, she is in perfect condition. 